\title{
Effect of Governance on Accelerating Literacy Rate to Ensure Sustainable Forestland: A Multiple Regression Analysis on Developing Countries
}

\author{
Tanbir Hossain \\ North Western University, Khulna, Department of Economics, 236, M.A. Bari Road, Sonadanga, \\ Khulna-9100, Bangladesh; tanbir_shovon_ku@yahoo.com
}

Received: 3 February 2021; Revised: 26 April 2021; Accepted: 4 May 2021; Published online: 24 May 2021

\begin{abstract}
Deforestation has been considered an alarming issue where every developing country focuses on accelerating the digit of GDP growth backlogging the environment quality badly. The literacy rate is a handy and effective tool that has a long-lasting impact on the economy; a good government body can launch a sustainable learning system that can enrich the practice of a green economy. This paper aims to investigate the joint effect of good governance and literacy rate on sustainable forestland in developing countries. The author uses World Bank secondary open data where the author applies a multiple regression model to analyze the effect on forestland. Due to the use of secondary data, there are some missing values where the author implies interpolation in R studio to imply regression Model. A sound and effective educational policy with government intervention can maintain a good balance among ecology, economy and socio-culture.
\end{abstract}

Key words: Forestland, deforestation, literacy rate, good governance, local people

Citation: Hossain, T. (2021). Effect of Governance on Accelerating Literacy Rate to Ensure Sustainable Forestland: A Multiple Regression Analysis on Developing Countries. Central European Journal of Geography and Sustainable Development, 3(1), 21-31. https://doi.org/10.47246/CEJGSD.2021.3.1.2 